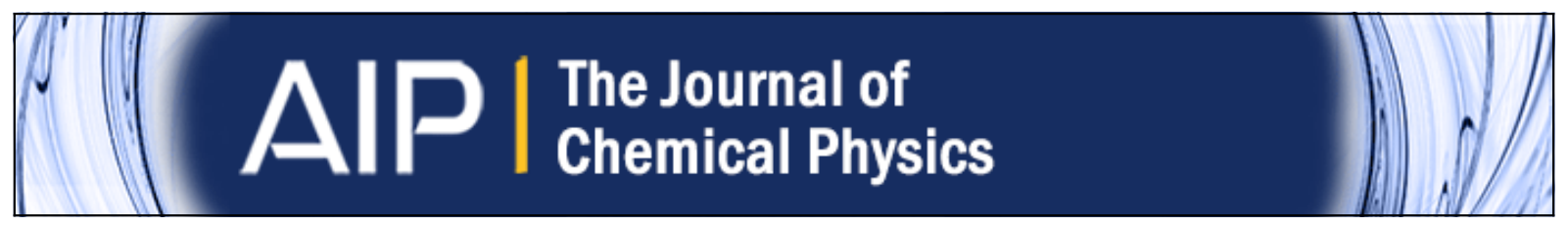

\title{
A hybrid perturbed-chain SAFT density functional theory for representing fluid behavior in nanopores: Mixtures
}

Gulou Shen, Xiaoyan Ji, Sven Öberg, and Xiaohua Lu

Citation: The Journal of Chemical Physics 139, 194705 (2013); doi: 10.1063/1.4825078

View online: http://dx.doi.org/10.1063/1.4825078

View Table of Contents: http://scitation.aip.org/content/aip/journal/jcp/139/19?ver=pdfcov

Published by the AIP Publishing

\section{Articles you may be interested in}

A hybrid perturbed-chain SAFT density functional theory for representing fluid behavior in nanopores

J. Chem. Phys. 138, 224706 (2013); 10.1063/1.4808160

Density-functional theory for polymer-carbon dioxide mixtures: A perturbed-chain SAFT approach

J. Chem. Phys. 137, 054902 (2012); 10.1063/1.4742346

A density functional theory for vapor-liquid interfaces using the PCP-SAFT equation of state

J. Chem. Phys. 131, 204705 (2009); 10.1063/1.3263124

Nonequilibrium molecular dynamics simulation of transport and separation of gases in carbon nanopores. II. Binary and ternary mixtures and comparison with the experimental data

J. Chem. Phys. 112, 910 (2000); 10.1063/1.480618

Nonequilibrium molecular dynamics simulation of transport and separation of gases in carbon nanopores. I.

Basic results

J. Chem. Phys. 111, 3252 (1999); 10.1063/1.479663

\section{AlP Re-register for Table of Content Alerts}




\title{
A hybrid perturbed-chain SAFT density functional theory for representing fluid behavior in nanopores: Mixtures
}

\author{
Gulou Shen, ${ }^{1,2}$ Xiaoyan $\mathrm{Ji},{ }^{1}$ Sven Öberg, ${ }^{3}$ and Xiaohua Lu ${ }^{2, a)}$ \\ ${ }^{1}$ Division of Energy Science/Energy Engineering, Luleå University of Technology, 97187 Luleå, Sweden \\ ${ }^{2}$ State Key Laboratory of Materials-Oriented Chemical Engineering, Nanjing University of Technology, \\ Nanjing 210009, China \\ ${ }^{3}$ Division of Material Science, Luleå University of Technology, 97187 Luleå, Sweden
}

(Received 20 July 2013; accepted 27 September 2013; published online 20 November 2013)

\begin{abstract}
The perturbed-chain statistical associating fluid theory (PC-SAFT) density functional theory developed in our previous work was extended to the description of inhomogeneous confined behavior in nanopores for mixtures. In the developed model, the modified fundamental measure theory and the weighted density approximation were used to represent the hard-sphere and dispersion free energy functionals, respectively, and the chain free energy functional from interfacial statistical associating fluid theory was used to account for the chain connectivity. The developed model was verified by comparing the model prediction with molecular simulation results, and the agreement reveals the reliability of the proposed model in representing the confined behaviors of chain mixtures in nanopores. The developed model was further used to predict the adsorption of methane-carbon dioxide mixtures on activated carbons, in which the parameters of methane and carbon dioxide were taken from the bulk PC-SAFT and those for solid surface were determined from the fitting to the pure-gas adsorption isotherms measured experimentally. The comparison of the model prediction with the available experimental data of mixed-gas adsorption isotherms shows that the model can reliably reproduce the confined behaviors of physically existing mixtures in nanopores. () 2013 AIP Publishing LLC. [http://dx.doi.org/10.1063/1.4825078]
\end{abstract}

\section{INTRODUCTION}

The properties of fluids confined in nanopores are different from those in the bulk phase due to geometrical restrictions and wall-molecule interactions. Understanding and prediction of equilibrium and dynamic properties of fluids and their mixtures in nanopores are of considerable importance in various industrial processes and scientific fields such as separation of mixtures by porous materials, wetting, lubrication, and tribology. ${ }^{1-3} \mathrm{~A}$ lot of methods have been used to represent the inhomogeneous behavior of confined fluids from experimental measurements, molecule simulations to theoretical models, and the classical density functional theory (DFT) in general provides reasonable description of inhomogeneous fluids with moderate computational cost. ${ }^{4}$

A variety of DFT models have been developed to predict the properties of inhomogeneous simple atomic and complex fluids, and those based on Wertheim's first order thermodynamic perturbation theory (TPT1) are promising. ${ }^{4-7}$ For example, Yu et al. ${ }^{8}$ developed a DFT based on Wertheim's TPT1 for chain conformation and bond orientation correlation function of hard-core multi-Yukawa chain fluids. Borouko et al. ${ }^{9}$ proposed a DFT to study the phase behavior of a two-component fluid in a slit-like pore with walls modified by tethered chains. These models accurately predict the microstructure of inhomogeneous fluid but it is infeasible to use such models to represent properties of real substances due to the fact that most of these models were applied for

\footnotetext{
a)Electronic mail: xhlu@ @jut.edu.cn
}

model molecules with integer segment numbers. Meanwhile, statistical associating fluid theory (SAFT)-based DFT models have been developed to account for the contribution to the free energy functional due to chain connectivity and site associating of inhomogeneous fluid. ${ }^{10-17}$ Among them, the models proposed by $\mathrm{Xu}$ et al. ${ }^{14} \mathrm{Hu}$ et al.,${ }^{15}$ Llovell et al. ${ }^{16}$ and Oliveria et $a l .{ }^{17}$ can be used to represent the interfacial properties where the external field is weak. For other models, little effort has been put into incorporating the long-range attraction perturbation that reduces to the SAFT EoS in the bulk limit.

In our previous work, ${ }^{18}$ a perturbed-chain (PC)-SAFTDFT model has been developed to represent the properties of fluid confined in nanopores. The developed PC-SAFTDFT model can reproduce the density profiles of chain fluids, and it is also feasible to represent the adsorption isotherms of physically existing substances, for example pure $\mathrm{CH}_{4}$ and $\mathrm{CO}_{2}$, on activated carbons. ${ }^{18}$ However, the extension of the developed model to mixtures has not yet been investigated.

In this work, the PC-SAFT-DFT model that has been developed in our previous work was extended in order to describe the property of mixtures confined in nanopores. The developed model will be used to predict the density profile of mixtures with "model" molecules and the prediction will be compared with the molecular simulation results for model verification. Moreover, the developed model will be used to predict the adsorption isotherms of methane-carbon dioxide mixtures on activated carbons to illustrate the model performance for real substances. 


\section{THEORY}

The model developed in our work was based on PCSAFT in which the hard-chain, dispersive, and associative interactions were considered. There are two versions of PCSAFT, one is for square-well chain molecule (first version) and the other is for modified square-well chain molecule (second version). ${ }^{19,20}$ In our work, the DFT model development was based on the second version of PC-SAFT, while the developed model can be easily extended to the first version of PC-SAFT.

For a mixture, we considered a chain mixture confined in a nanopore, and chain $i$ was formed of $m_{i}$ segments with a diameter $\sigma_{i}$. Following this consideration, in DFT, for an open system, the grand potential $\Omega$ at fixed $\mu_{i}, V$, and $T$ is given by the equation

$$
\Omega\left[\rho_{i}(\boldsymbol{r})\right]=A\left[\rho_{i}(\boldsymbol{r})\right]-\sum_{i} \int d \boldsymbol{r}^{\prime} \rho_{i}\left(\boldsymbol{r}^{\prime}\right)\left(\mu_{i}-m_{i} V_{i, e x t}\left(\boldsymbol{r}^{\prime}\right)\right),
$$

where $A$ is the Helmholtz free energy, $\rho_{i}(\boldsymbol{r})$ is the molecular density of component $i, \mu_{i}$ is the chemical potential, $m_{i}$ is the number of segments in a chain for component $i$, and $V_{i, \text { ext }}(\boldsymbol{r})$ is the external field acting on the segment of component $i$.

Following PC-SAFT EoS, the Helmholtz free energy $A$ can be expressed as

$$
\begin{aligned}
A\left[\rho_{i}(\boldsymbol{r})\right]= & A^{i d}\left[\rho_{i}(\boldsymbol{r})\right]+A^{h s}\left[\rho_{i}(\boldsymbol{r})\right]+A^{\text {chain }}\left[\rho_{i}(\boldsymbol{r})\right] \\
& +A^{\text {disp }}\left[\rho_{i}(\boldsymbol{r})\right]+A^{\text {assoc }}\left[\rho_{i}(\boldsymbol{r})\right],
\end{aligned}
$$

where $A^{\text {id }}$ is the ideal free energy, $A^{\text {hs }}, A^{\text {chain }}, A^{\text {disp }}$, and $A^{\text {assoc }}$ are the excess free energies due to hard sphere, chain, dispersive and associative interactions, respectively. The extension of PC-SAFT to inhomogeneous mixed-fluids is described in the following part.

\section{A. Ideal and hard chain terms}

Following our previous work, ${ }^{18}$ the model developed by Tripathi and Chapman ${ }^{7}$ was used to represent the ideal and chain terms. The reference ideal free energy is written as the free energy of the ideal atomic gas mixture

$$
\beta A^{i d}\left[\rho_{i}\right]=\sum_{i} \int d \boldsymbol{r} m_{i} \rho_{i}(\boldsymbol{r})\left(\ln \rho_{i}(\boldsymbol{r})-1\right),
$$

where $\beta=1 / k T$ and $k$ is the Boltzmann constant. The hard sphere free energy was calculated using the modified fundamental measure theory and given by ${ }^{21}$

$$
\beta A^{h s}\left[\rho_{i}\right]=\int d \boldsymbol{r} \Phi^{h s}\left[n_{\alpha}(\boldsymbol{r})\right] .
$$

The expression of $\Phi^{\text {hs }}$ is exactly the same as those for pure fluid ${ }^{18}$ but with Rosenfeld weighted densities $n_{\alpha}(\boldsymbol{r})$ for mixtures calculated with

$$
n_{\alpha}(\boldsymbol{r})=\sum_{i} n_{\alpha, i}(\boldsymbol{r})=\sum_{i} m_{i} \int d \boldsymbol{r}^{\prime} \rho_{i}\left(\boldsymbol{r}^{\prime}\right) w_{i}^{\alpha}\left(\boldsymbol{r}-\boldsymbol{r}^{\prime}\right),
$$

where $w^{\alpha}$ represents weight functions, i.e., four scalar and two vector functions, and the expressions have been described in our previous work. ${ }^{18}$

The chain free energy functional is calculated based on the approach developed by Tripathi and Chapman, ${ }^{7}$ and for mixture it is given by

$$
\begin{aligned}
\beta A^{\text {chain }}[\rho]= & \sum_{i}-\left(m_{i}-1\right) \int d \boldsymbol{r} \rho_{i}(\boldsymbol{r})\left[\operatorname { l n } \left\{y_{i i}^{c o n t}\left[\bar{\rho}_{i}(\boldsymbol{r}), d_{i}\right]\right.\right. \\
& \left.\left.\times \int d \boldsymbol{r}^{\prime} \frac{\delta\left(\left|\boldsymbol{r}-\boldsymbol{r}^{\prime}\right|-d_{i}\right)}{4 \pi d_{i}^{2}} \rho_{i}\left(\boldsymbol{r}^{\prime}\right)\right\}-1\right]
\end{aligned}
$$

where $y_{i i}^{c o n t}\left[\bar{\rho}_{i}(\boldsymbol{r}), d_{i}\right]$ is the value of cavity correlation function at contact, and it is given by

$$
y_{i i}^{c o n t}\left[\bar{\rho}_{i}(\boldsymbol{r}), d_{i}\right]=\frac{1}{1-\bar{\zeta}_{3}}+\frac{3 d_{i} \bar{\zeta}_{2}}{2\left(1-\bar{\zeta}_{3}\right)^{2}}+\frac{d_{i}^{2}\left(\bar{\zeta}_{2}\right)^{2}}{2\left(1-\bar{\zeta}_{3}\right)^{3}},
$$

and $\bar{\zeta}_{k}=\sum_{i} \frac{\pi}{6} m_{i} d_{i}^{k} \bar{\rho}_{i}(\boldsymbol{r})$, which is evaluated at the "coarsegrained density," i.e.,

$$
\bar{\rho}_{i}(\boldsymbol{r})=\frac{3}{4 \pi d_{i}^{3}} \int_{\left|\boldsymbol{r}-\boldsymbol{r}^{\prime}\right|<d_{i}} d \boldsymbol{r}^{\prime} \rho_{i}\left(\boldsymbol{r}^{\prime}\right) \text {. }
$$

In Eqs. (7) and (8), $d_{i}$ is the temperature-dependent segment diameter of component $i$ calculated with $d_{i}(T)$ $=\sigma_{i}\left[1-0.12 \exp \left(-3 \varepsilon_{i f} / k T\right)\right]$ where $\varepsilon_{i f}$ is the energy parameter of fluid-fluid interaction and $\sigma_{i}$ is the segment diameter of molecule.

\section{B. Dispersion term}

Following our previous work, ${ }^{18}$ a weighted density approximation was used to represent the contribution of dispersive attraction to free energy for mixed-fluids. In this approximation the dispersion free energy functional is given by

$$
\beta A^{d i s p}\left[\rho_{i}\right]=\sum_{i} \sum_{j} \int d \boldsymbol{r} \rho_{i}(\boldsymbol{r}) \bar{\rho}_{j, d i s p}(\boldsymbol{r}) f\left(\bar{\eta}_{d i s p}(\boldsymbol{r})\right)
$$

where

$$
\begin{aligned}
& f\left(\bar{\eta}_{d i s p}(\boldsymbol{r})\right) \\
&=-2.0 \pi m_{i} m_{j} I_{1}\left(\bar{\eta}_{d i s p}(\boldsymbol{r}), \bar{m}\right) \frac{\varepsilon_{i j}}{k T} \sigma_{i j}^{3} \\
&-\pi m_{i} m_{j} \bar{m} C_{1}\left(\bar{\eta}_{d i s p}(\boldsymbol{r}), \bar{m}\right) I_{2}\left(\bar{\eta}_{d i s p}(\boldsymbol{r}), \bar{m}\right)\left(\frac{\varepsilon_{i j}}{k T}\right)^{2} \sigma_{i j}^{3},
\end{aligned}
$$

with

$$
\begin{aligned}
C_{1}\left(\bar{\eta}_{d i s p}(\boldsymbol{r})\right)= & 1+\bar{m} \frac{8 \bar{\eta}_{d i s p}(\boldsymbol{r})-2 \bar{\eta}_{d i s p}(\boldsymbol{r})^{2}}{\left(1-\bar{\eta}_{d i s p}(\boldsymbol{r})\right)^{4}} \\
& +(1-\bar{m}) \frac{20 \bar{\eta}_{d i s p}(\boldsymbol{r})-27 \bar{\eta}_{d i s p}(\boldsymbol{r})^{2}+12 \bar{\eta}_{d i s p}(\boldsymbol{r})^{3}-2 \bar{\eta}_{d i s p}(\boldsymbol{r})^{4}}{\left[\left(1-\bar{\eta}_{d i s p}(\boldsymbol{r})\right)\left(2-\bar{\eta}_{d i s p}(\boldsymbol{r})\right)\right]^{2}}
\end{aligned}
$$


and

$$
\left\{\begin{array}{c}
\bar{m}=\frac{\sum_{i} m_{i} \bar{\rho}_{i, d i s p}(\boldsymbol{r})}{\sum_{i} \bar{\rho}_{i, d i s p}(\boldsymbol{r})} \\
\bar{\eta}_{\text {disp }}(\boldsymbol{r})=\frac{\pi}{6} \sum_{i} m_{i} d_{i}^{3} \bar{\rho}_{i, d i s p}(\boldsymbol{r})
\end{array} .\right.
$$

The Berthelot-Lorentz combining rules were used to determine the size and energy parameter of unlike fluid-fluid interaction,

$$
\left\{\begin{array}{l}
\sigma_{i j}=\frac{\sigma_{i}+\sigma_{j}}{2} \\
\varepsilon_{i j}=\left(1-k_{i j}\right) \sqrt{\varepsilon_{i} \varepsilon_{j}}
\end{array},\right.
$$

where $k_{i j}$ is the binary interaction parameter. ${ }^{20}$

In Eqs. (10) and (11a), $\bar{\eta}_{\text {disp }}(\boldsymbol{r})$ is the average packing fraction, in which the weighted density is given by

$$
\bar{\rho}_{j, d i s p}(\boldsymbol{r})=\int d \boldsymbol{r}^{\prime} \rho_{j}\left(\boldsymbol{r}^{\prime}\right) w_{j, d i s p}\left(\boldsymbol{r}-\boldsymbol{r}^{\prime}\right),
$$

where $w_{j, d i s p}(\boldsymbol{r})$ is the weight function and the detailed information is described in Sec. II D.

\section{Association term}

The approach proposed by $\mathrm{Yu}$ and $\mathrm{Wu}^{11}$ was used to represent the association term and the free energy functional due to association is given by

$$
\beta A^{a s s o c}[\rho]=\int d \boldsymbol{r} \Phi^{a s s o c}\left[n_{\alpha}(\boldsymbol{r})\right],
$$

where

$$
\begin{aligned}
\Phi^{a s s o c}\left[n_{\alpha}(\boldsymbol{r})\right]= & \sum_{i} \sum_{j=1}^{s} S_{i}^{j}\left(\frac{n_{2, i}^{2}-\boldsymbol{n}_{v 2, i} \cdot \boldsymbol{n}_{v 2, i}}{\pi d_{i}^{2} n_{2, i}}\right) \\
& \times\left[\ln X_{i}^{j}(\boldsymbol{r})-\frac{X_{i}^{j}(\boldsymbol{r})}{2}+\frac{1}{2}\right] .
\end{aligned}
$$

In Eq. (14), $s$ is the number of types of association sites, $S_{i}^{j}$ is the number of association sites of type $j$ of component $i$, and $X_{i}^{j}$ is the fraction of component $i$ not bonded at site $j$ and calculated using the mass action equation:

$$
X_{i}^{j}=\frac{1}{1+\sum_{k} \sum_{l=1, l \neq j}^{s} S_{k}^{l}\left(\frac{n_{2, k}^{2}-\boldsymbol{n}_{v 2, k} \cdot \boldsymbol{n}_{v 2, k}}{\pi d_{k}^{2} n_{2, k}}\right) X_{k}^{l}(\boldsymbol{r}) \Delta_{i k}^{j l}(\boldsymbol{r})} .
$$

\section{Weight function and PC-SAFT}

As mentioned in the foregoing text, there are two versions of PC-SAFT, i.e., square-well chain molecule (first version) and modified square-well chain molecule (second version). In the first version of PC-SAFT, it is a rigorous square-well potential with a square-well length $\lambda=1.5$ and the segment diameter is a constant. For the second version of PC-SAFT, it is neither a rigorous square-well potential nor a Lennard-Jones
(LJ) potential, and the hard sphere diameter is temperaturedependent. For the bulk PC-SAFT, the different potential utilization leads to two sets of constants calculating dispersive interaction and the conversion between two versions of PCSAFT is on the hard sphere diameter and the set of constants in calculating dispersive interaction. However, for the developed PC-SAFT-DFT, the weight function in Eq. (12) also depends on the choice of potential, and thus the effect of the weight function as well as the version of PC-SAFT on the developed PC-SAFT-DFT model will be further discussed in this section.

For the first version of PC-SAFT or the rigorous squarewell potential molecules, the weight function can be obtained from the method proposed by Ye et al. ${ }^{22}$ and the weighted density is an integral within the range of interaction, i.e.,

$$
\left\{\begin{array}{l}
w_{j, d i s p}(\boldsymbol{r})=\frac{3 \theta\left(\lambda \sigma_{j}-r\right)}{4 \pi\left(\lambda \sigma_{j}\right)^{3}} \\
\bar{\rho}_{j, d i s p}(\boldsymbol{r})=\frac{3}{4 \pi\left(\lambda \sigma_{j}\right)^{3}} \int_{\left|\boldsymbol{r}-\boldsymbol{r}^{\prime}\right|<\lambda \sigma_{j}} d \boldsymbol{r}^{\prime} \rho_{j}\left(\boldsymbol{r}^{\prime}\right)
\end{array}\right.
$$

In Eq. (16), $\lambda \sigma_{j}$ represents the weighting distance and the square-well length $\lambda$ equals to 1.5 for the first version of PC-SAFT.

For the second version of PC-SAFT, if it is assumed as a square-well potential in the DFT model development, the calculation of weight function and the weighted density will be exactly the same as that expressed in Eq. (16). As the squarewell length is needed for integral, it was assumed to be 1.5 in our work after the preliminary study. If the second version of PC-SAFT is assumed as a LJ potential, there are several ways to calculate the weight function, i.e., the normalized mean field weight function, ${ }^{23,24}$ the step function with a temperature-dependent diameter ${ }^{25}$ or the normalized weight function calculated from direct correlation function. ${ }^{26}$ In this work, we chose a common normalized mean field weight function due to its simplicity and known performance, and then the weighted density is an integral within the range of attraction, ${ }^{23,24}$ i.e.,

$$
w_{d i s p}(\boldsymbol{r})=u_{a t t r}(\boldsymbol{r}) / \int d \boldsymbol{r} u_{a t t r}(\boldsymbol{r}) .
$$

In Eq. (17), $u_{\text {attr }}(\boldsymbol{r})$ is the $\mathrm{LJ}$ attractive potential and the LJ cutoff distance was set as $4 \sigma_{i}$ after the preliminary study.

The effect of the weight function on the model performance is discussed in Sec. III.

\section{E. Numerical procedure}

Minimization of the grand potential with respect to the density profile of component $i$ yields the following EulerLagrange equation:

$$
\frac{\delta \Omega[\rho(\boldsymbol{r})]}{\delta \rho_{i}(\boldsymbol{r})}=\frac{\delta A[\rho(\boldsymbol{r})]}{\delta \rho_{i}(\boldsymbol{r})}-\left(\mu_{i}-m_{i} V_{i, e x t}(\boldsymbol{r})\right)=0 .
$$

At equilibrium, $\mu_{i}$ is equal to the bulk chemical potential $\mu_{i, b}$ that is calculated from the temperature, pressure, and homogeneous composition. The equilibrium density profile 
can be solved using simple Picard iteration. In a planar geometry, the three-dimensional integral is reduced to a onedimensional integral.

\section{RESULTS AND DISCUSSION}

Using the developed model, the density profile and adsorption isotherm can be obtained for both "model" molecules and physically existing substances. Meanwhile, using the molecular simulation, the density profiles for hard-chain mixtures and square-well chain mixtures have been simulated. In addition, the adsorption isotherms of methane-carbon dioxide mixtures on activated carbons have been measured experimentally. Therefore, in this work, in order to verify the model performance, the developed model was firstly used to predict the density profiles with the parameters set in molecular simulations, and the model prediction was compared with molecular simulation results. To illustrate the model performance for real substances besides "model" molecules, the model was further used to predict the adsorption isotherms of methanecarbon dioxide mixtures on activated carbons in which the parameters describing the external force were obtained from the fitting of the experimental adsorption data of pure gas.

As mentioned in the foregoing text, the developed DFT model is based on the second version of PC-SAFT. However, most of the results obtained in the molecular simulation were obtained with the assumption of square-well fluids with $\lambda=1.5$, which is the case of the first version of PC-SAFT. Therefore, in the part of model verification, in order to compare the DFT model predictions with molecular simulation results, the DFT model developed in this work was extended to the first version of PC-SAFT by replacing the temperaturedependent segment diameter with a fixed segment diameter and the set of constants for calculating dispersive interaction as well as the weighted density calculated by Eq. (16).

In the part of the model representation of gas adsorption isotherms for real substances, the developed model on the basis of the second version of PC-SAFT was used, and the weighted density was calculated with two options, i.e., Eqs. (16) and (17), respectively. In addition, a LJ potential was used to describe the interaction between surface and fluid, and the parameters describing the external force were obtained from the fitting of the experimental pure-gas adsorption data by assuming pores with an average width. Then the effect of using a pore size distribution (PSD) on the model performance was discussed.

\section{A. Density profiles of hard chain mixtures in a hard slit}

The hard-chain mixture confined in a hard slit has been studied by Tripathi and Chapman, ${ }^{7}$ while the chain free energy functional for mixtures was not given explicitly in the literature. To validate the hard chain term for mixtures developed in this work, the density profiles predicted by the developed model were compared with Monte Carlo (MC) simulation results. The system parameters (interaction, parameters of size and surface-fluid interaction as well as density) were

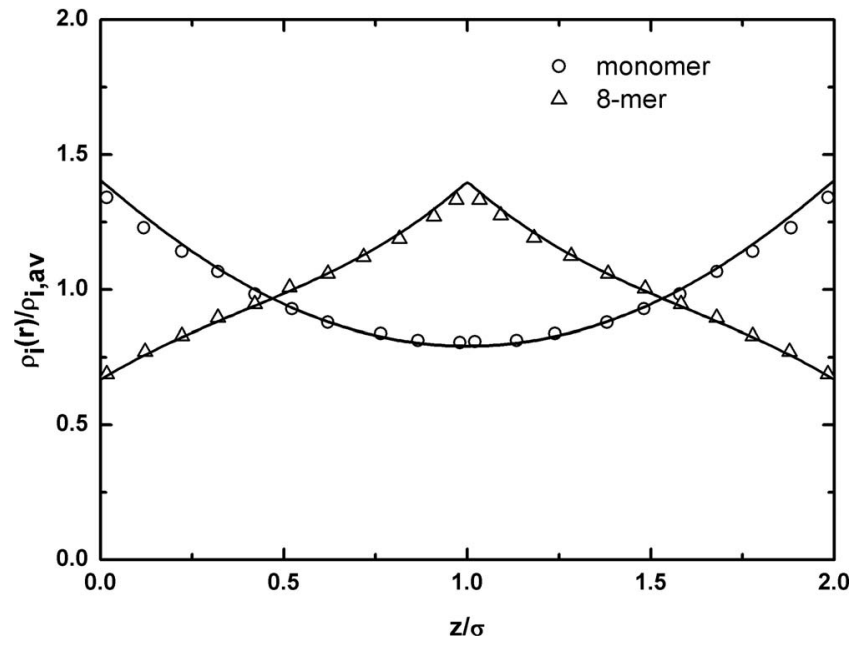

FIG. 1. Density profiles of monomer and 8-mer hard-chain mixture with monomer mole fraction 0.5 at $\eta_{a v}=0.12$. The slit width is $H=2 \sigma$. Lines: DFT model predictions; symbols: MC simulation data. ${ }^{27}$

set to be exactly the same as those in molecular simulations. The surface-fluid interaction is given by

$$
V_{\text {ext }}(z)=\left\{\begin{array}{cc}
\infty & z<0 \\
0 & z>0
\end{array},\right.
$$

where $z$ is the perpendicular distance between fluid and surface.

In prediction, $\eta_{a v}$ is the average packing fraction ( $\eta_{a v}=\sum_{i} \frac{\pi}{6} m_{i} \sigma^{3} \rho_{i, a v}$ where $\rho_{i, a v}$ is the average density in the pore $\left(=\frac{1}{H} \int_{0}^{H} d z \rho_{i}(z), H\right.$ is the width of the slit)). Figure 1 shows the model predictions of the density profiles of monomer and 8-mer chain mixture confined in a hard slit. The mole fraction of monomer is $0.5\left(\eta_{a v, 1}=\eta_{a v, 2}=0.06\right)$, and the width of the slit is $H=2 \sigma$. In this case, the monomer density decrease monotonically away from the wall, while the chain density increase and reach the maximum at the pore center. The developed model accurately captures the enhancement of monomer and depletion of 8-mer near the surface compared with the Monte Carlo simulation results. ${ }^{27}$

Figure 2 displays the segment density profiles of 3-mer and 12-mer chains mixtures confined in hard slit at average packing fraction $0.1,0.2$, and 0.3 predicted by the developed model and results from molecular simulations. ${ }^{28}$ The mole fraction of 3-mer is 0.5 and the width of the slit is $H=10 \sigma$. At low density the chains are depleted near the surface and long chains are more depleted than short chains. At high density the chains are enhanced near the surface and short chains are more enhanced. The model predictions are in good agreement with simulation results at low density. The densities near the surface show deviations from simulation results at high density.

As mentioned that Tripathi and Chapman ${ }^{7}$ have developed a DFT model for representing the hard-chain mixture confined in a hard slit but the expression for mixtures was not given explicitly in the literature. The predictions of the model developed in this work were compared with those in the work by Tripathi and Chapman. ${ }^{7}$ The comparison shows good agreement for most of systems predicted by these two 

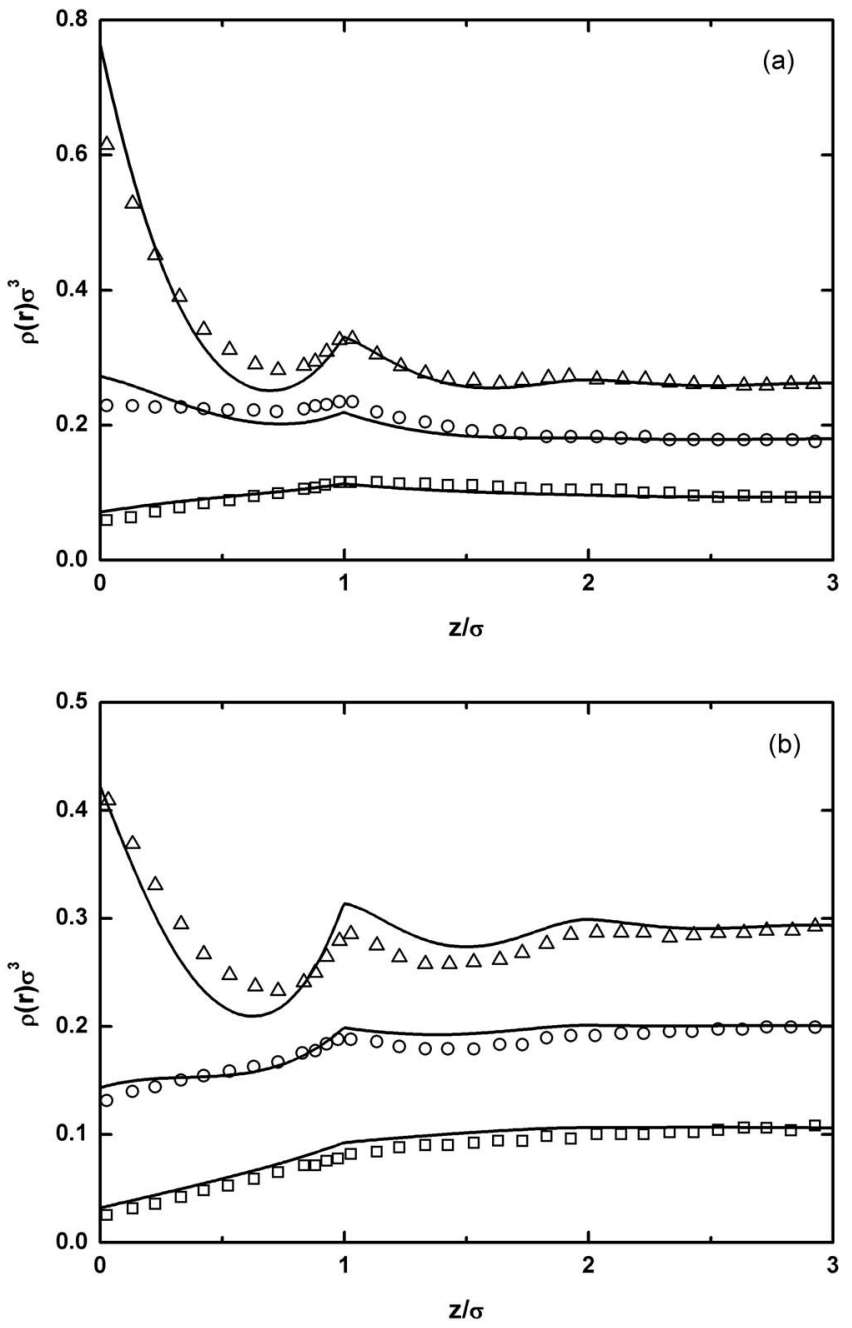

FIG. 2. Segment density profiles of 3-mer (a) and 12-mer (b) hard chains confined in a hard slit in 3-mer and 12-mer mixtures with 3-mer mole fraction 0.5. Lines: DFT model predictions; symbols: MC simulation data, ${ }^{28}$ $\triangle: \eta_{a v}=0.3 ; \bigcirc: \eta_{a v}=0.2 ; \square: \eta_{a v}=0.1$.

models while for some cases there are some discrepancies, which implies that the expression for mixtures should not be exactly the same.

\section{B. Density profiles of square-well chain mixtures in slit pores}

The developed model was used to predict the density profiles of square-well chain mixtures in a slit pore with width $H=10 \sigma$. The interaction between hard surface and fluid represented is given by Eq. (19). The interaction between squarewell surface and fluid is expressed as in Eq. (20):

$$
V_{\text {ext }}(z)= \begin{cases}\infty & z<0 \\ -\varepsilon_{w} / k T & 0 \leq z<\lambda_{w} \sigma \\ 0 & z \geq \lambda_{w} \sigma\end{cases}
$$

where $z$ is the perpendicular distance between fluid and surface. The surface-fluid interaction parameter is $\lambda_{w}=1$ and $\varepsilon_{w} / k T=0.1$. The temperature is $k T / \varepsilon_{f}=6.0$.
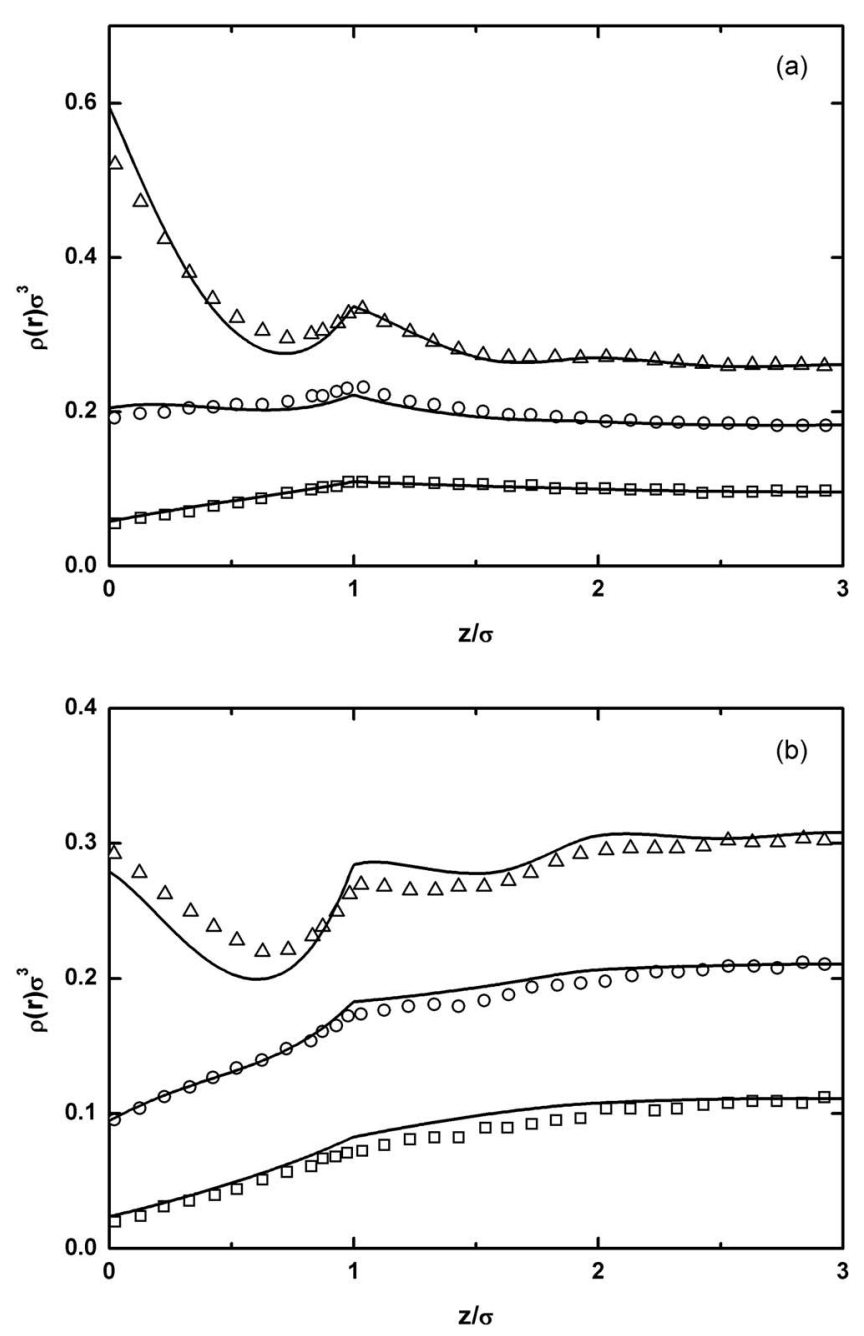

FIG. 3. Segment density profiles of 3-mer (a) and 12-mer (b) square-well chains confined in a hard slit in 3-mer and 12-mer mixtures with 3-mer mole fraction 0.5. Lines: DFT model predictions; symbols: $\mathrm{MC}$ simulation data, ${ }^{28}$ $\triangle: \eta_{a v}=0.3 ; \bigcirc: \eta_{a v}=0.2 ; \square: \eta_{a v}=0.1$.

Figure 3 displays the segment density profiles of 3-mer and 12-mer square-well chain mixtures confined in a hard slit at three different average packing fractions, i.e., 0.1, 0.2, and 0.3 . The mole fraction of 3 -mer is 0.5 . The density profiles are similar to those in Figure 2. At high density the chains are enhanced near the surface. Short chains show stronger enhancement than long chain and have a maximum density on the surface. At low density chains are depleted near the surface. The density profiles increase monotonous and have a cusp at about $z=\sigma$.

The model predictions were further compared with simulation data, ${ }^{28}$ at low density the predictions are in good agreement with Monte Carlo simulation results. There are some discrepancies at high density, which is partially due to the performance of hard chain term as shown in Figure 2.

Figure 4 displays the segment density profiles of 3-mer and 12-mer square-well chain mixtures confined in a squarewell slit. The density discontinuities occur as expected at $z=\sigma$ when the surface-fluid interaction is discontinuous. The predictions show the same agreement with Monte Carlo simulation results ${ }^{28}$ as those in Figure 3. 

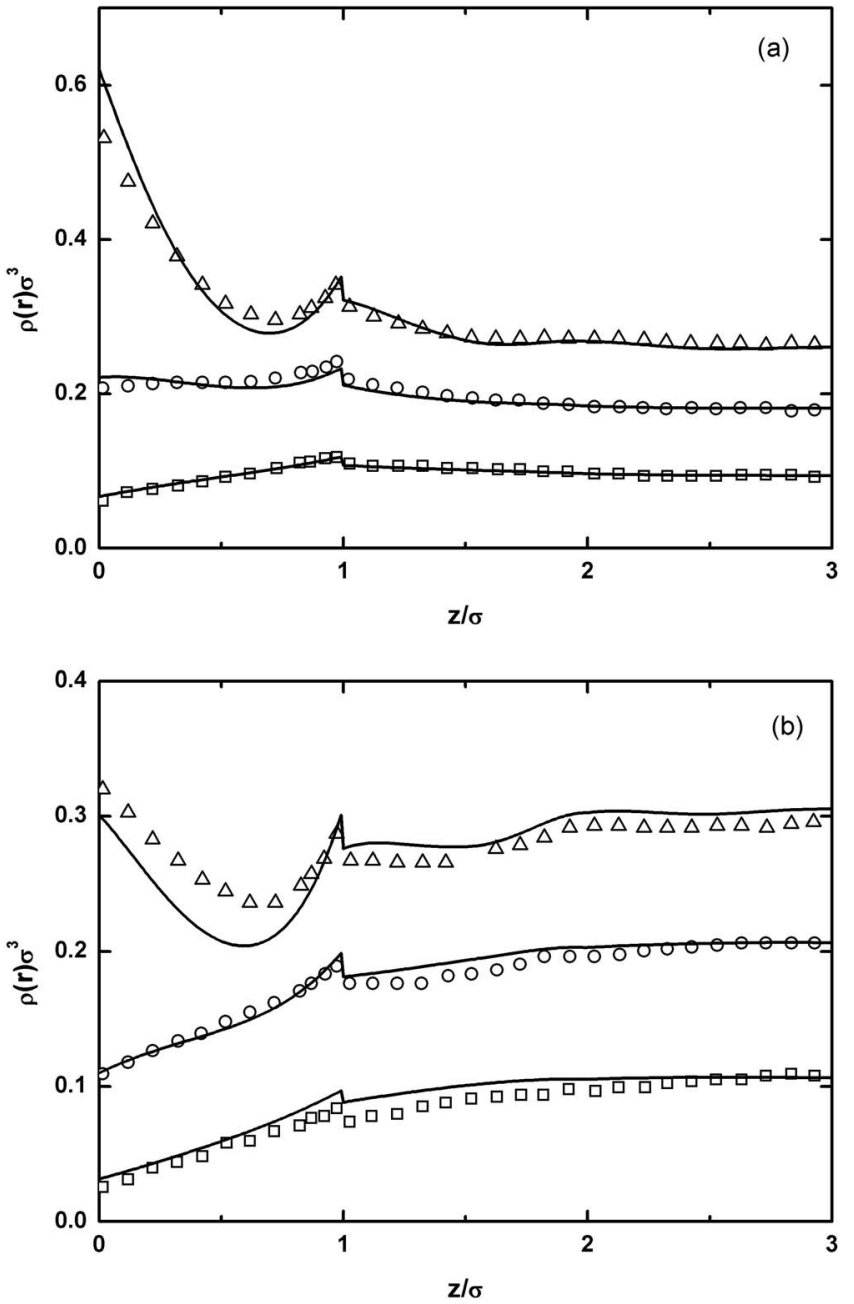

FIG. 4. Segment density profiles of 3-mer (a) and 12-mer (b) square-well chains confined in a square-well slit in 3-mer and 12-mer mixtures with 3-mer mole fraction 0.5. Lines: DFT model predictions; symbols: MC simulation data, $^{28} \triangle: \eta_{a v}=0.3 ; \bigcirc: \eta_{a v}=0.2 ; \square: \eta_{a v}=0.1$.

\section{Prediction of adsorption of gas mixtures}

In the foregoing sections, the prediction of the developed model was verified by comparing with the molecular simulation results, and the agreement indicates the model's reliability for "model" molecules. In this section we investigated the model performance for real substances.

The gas adsorption of small molecules such as methane $\left(\mathrm{CH}_{4}\right)$ and carbon dioxide $\left(\mathrm{CO}_{2}\right)$ on nanoporous materials has been widely studied experimentally, and the adsorption isotherm has been measured extensively for the purpose of separating $\mathrm{CO}_{2}$ from gas mixture or selective exchange adsorption of $\mathrm{CO}_{2}$ from $\mathrm{CH}_{4}$ for enhanced coal-bed methane. To illustrate the developed DFT model performance, the adsorption of pure methane and carbon dioxide and binary mixtures on Calgon F400 activated carbon was studied as an example in this work.

In general, the material has a wide range of pore size distribution and shape. In order to reduce the computation complexity, in modeling, we assumed that the gas molecule was adsorbed in a single slit-like pore with an average width. The solid-fluid interaction $V_{\text {ext }}(z)$ was represented by Lee's $10-4$
TABLE I. Model parameters for molecules of $\mathrm{CH}_{4}$ and $\mathrm{CO}_{2}$.

\begin{tabular}{llll}
\hline \hline Substance & \multicolumn{1}{c}{$\mathrm{m}$} & $\sigma_{f}(\AA)$ & $\varepsilon_{f} / k(\mathrm{~K})$ \\
\hline $\mathrm{CH}_{4}$ & 1.0 & 3.7039 & 150.03 \\
$\mathrm{CO}_{2}$ & 2.0729 & 2.7852 & 169.21 \\
\hline \hline
\end{tabular}

Lennard-Jones potential, ${ }^{29}$ which is given by

$$
\begin{aligned}
V_{\text {ext }, i}(z)= & 2 \pi \rho_{\text {atom }} \varepsilon_{s i} \sigma_{s i}^{2} \\
& \times\left[\frac{2}{5}\left(\frac{\sigma_{s i}}{z}\right)^{10}-\sum_{i=1}^{4} \frac{\sigma_{s i}^{4}}{(z+(i-1) \Delta)^{4}}\right],
\end{aligned}
$$

where $\rho_{\text {atom }}$ is the solid atom density, $\sigma_{s i}$ is the solid-fluid diameter, $\Delta$ is the carbon interplanar distance, and $\varepsilon_{s i}$ is the potential representing the interaction between surface and fluid segment.

The excess adsorption $(q)$ of component $i$ in mixture is calculated by

$$
q_{i}=\frac{A_{i}^{*}}{2} \int_{\sigma_{s i}}^{H-\sigma_{s i}} d z\left(\rho_{i}(z)-\rho_{b u l k, i}\right),
$$

where $A_{i}^{*}$ is the effective surface area of the adsorbent and $H$ is the width of the slit.

To represent gas adsorptions, the properties of fluid and solid surface need to be provided. In this work, the parameters of fluid molecules were taken from the literature, ${ }^{20}$ which were obtained from the fitting of the experimental data in the bulk phase. The parameters for $\mathrm{CH}_{4}$ and $\mathrm{CO}_{2}$ used in this work are listed in Table I, and the binary interaction parameter is $0.065 .^{20}$

The parameters of solid (the wall) can be adjustable or estimated. In order to decrease the number of adjustable parameters, in this work, some parameters of solid were taken from literatures, ${ }^{29}$ i.e., for Calgon $\mathrm{F} 400$ activated carbon, the solid atom density $\rho_{\text {atom }}$ was set to be 0.382 atoms $/ \AA^{2}$, and both $\Delta$ and $\sigma_{s}$ were set to be $3.35 \AA$. In addition, the following mixing rule was used to obtain $\sigma_{s i}$ and $\varepsilon_{s i}$.

$$
\begin{gathered}
\sigma_{s i}=\frac{\sigma_{s}+\sigma_{i}}{2}, \\
\varepsilon_{s i}=\sqrt{\varepsilon_{s} \varepsilon_{i}} .
\end{gathered}
$$

The adsorption quantity calculated by the model is sensitive to the value of slit width $H$. As $H$ was assumed to be the average of pore width, in this work $H$ was considered as an adjustable parameter but set to be a fixed value for the same porous materials, and then it is independent of the adsorbed gas. The surface area $A_{i}^{*}$ was specified as an adjustable parameter for each component. $\varepsilon_{s}$ was set as one more adjustable parameter for one system. Therefore, in this work, there are 4 adjustable parameters in total in order to represent the adsorption of methane and carbon dioxide on Calgon F400 activated carbons, i.e., the size of pore $H$, two surface areas $A_{i}^{*}$ and $\mathrm{LJ}$ interaction parameter $\varepsilon_{s}$. These adjustable parameters were obtained from the fitting of experimental 
TABLE II. DFT model parameters for representing $\mathrm{CH}_{4}$ and $\mathrm{CO}_{2}$ adsorption on Calgon F400 activated carbon.

\begin{tabular}{lccccc}
\hline \hline & & & \multicolumn{2}{c}{$A\left(\mathrm{~m}^{2} / \mathrm{g}\right)$} & \\
\cline { 3 - 4 } & $\mathrm{H}(\AA)$ & $\varepsilon_{s} / k(\mathrm{~K})$ & $\mathrm{CH}_{4}$ & $\mathrm{CO}_{2}$ & ARD $(\%)$ \\
\hline Case 1 & 13.5 & 30.20 & 887.9 & 778.5 & 7.5 \\
Case 2 & 13.4 & 39.08 & 953.8 & 990.1 & 5.0 \\
\hline \hline
\end{tabular}

data of pure gas adsorption isotherms for both $\mathrm{CH}_{4}$ and $\mathrm{CO}_{2}$ on Calgon F400 activated carbon. The average relative deviation $\left(A R D=\frac{100}{N_{\exp }} \sum_{i=1}^{N^{\exp }}\left|\frac{q_{i}^{c a l}-q_{i}^{\text {exp }}}{q_{i}^{\text {exp }}}\right|\right)$ was used to illustrate the model performance, where $N_{\text {exp }}$ is the number of experimental data points, $q_{i}^{c a l}$ is the calculated excess adsorption value, and $q_{i}^{\text {exp }}$ is the experimental excess adsorption value.

Using the experimental data measured by Sudibandriyo and co-workers at $318.2 \mathrm{~K},{ }^{30}$ the DFT model parameters were obtained and listed in Table II with the ARD 7.5\%. Both the model results and experimental data are shown in Figure 5 (Case 1) with reasonable agreements.

The model was used to predict the adsorption for gas mixture without any additional parameters. Figure 6 (Case 1) shows the comparison of the predicted adsorption isotherms of mixtures with the experimental data. The model captures the maxima in the isotherms. The prediction is better for systems with higher $\mathrm{CH}_{4}$ composition and this is partially because the lower deviation of the correlation for pure $\mathrm{CH}_{4}$ adsorption isotherm. The deviation of model prediction for mixed-gas adsorption is listed in Table III.

It should be pointed out that the model results depend on the weight function as shown in Eq. (12). For the developed model, the weight function can be two options: one is based on square-well potential and the other is based on LJ potential. Up to now, the results illustrated in this section are the model results based on square-well potential, i.e., the weight function is calculated with Eq. (16) and the square-well length

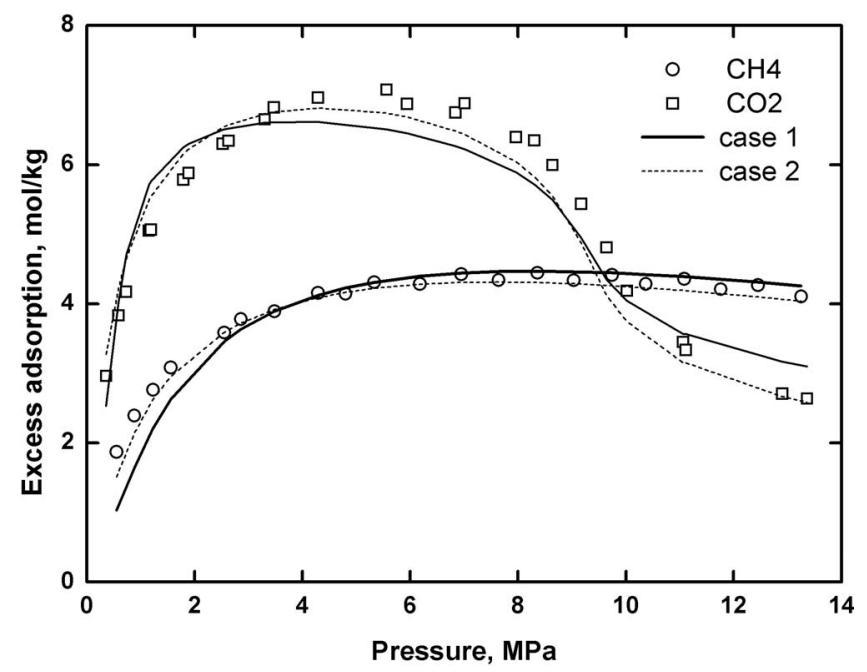

FIG. 5. Methane and carbon dioxide adsorption on Calgon F400 activated carbon. Symbols are experimental data; ${ }^{30}$ lines represent the results of the model. Case 1 represents the results of Eq. (16); Case 2 represents the results of Eq. (17).
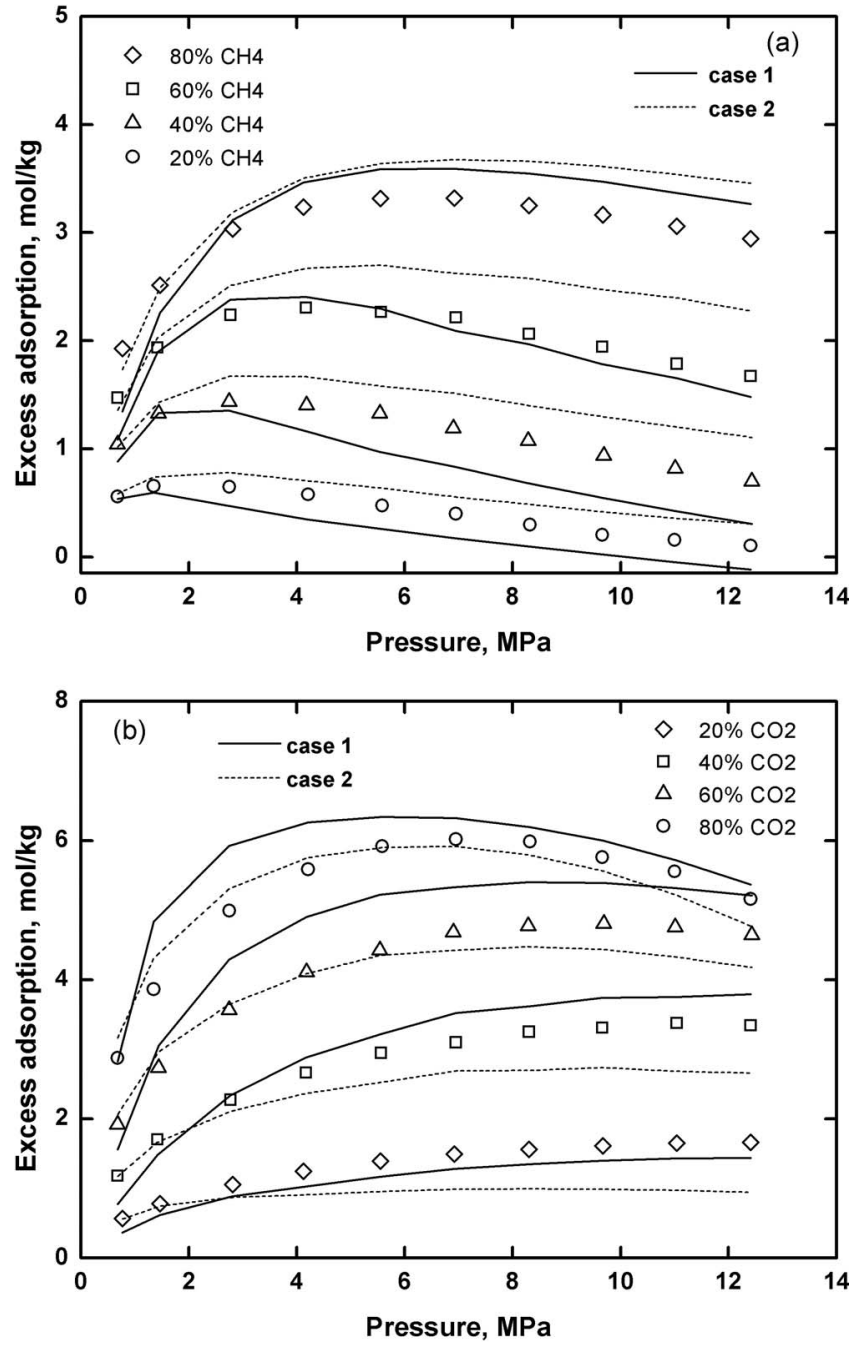

FIG. 6. Excess adsorption of $\mathrm{CH}_{4}$ (a) and $\mathrm{CO}_{2}$ (b) in $\mathrm{CH}_{4} / \mathrm{CO}_{2}$ mixtures on Calgon F400 activated carbon at $318.2 \mathrm{~K}$. Symbols are experimental data; ${ }^{30}$ lines represent the results of the model. The $\mathrm{CH}_{4}$ feed composition is $\diamond: 80 \%$; $\square: 60 \% ; \triangle: 40 \%$; $\bigcirc: 20 \%$. Case 1 represents the results of Eq. (16); Case 2 represents the results of Eq. (17).

$\lambda$ was set to be 1.5 . To further investigate the effect of weight function choice on model performance, the model based on LJ potential was checked and the results are summarized in the follow paragraph.

If the LJ potential in perturbation term was chosen for PC-SAFT-DFT model development, the weight function (Eq. (17)) was used to obtain the weighted density, and then the model was used to represent the mixed-gas adsorption isotherms for the same systems. The model parameters and the average relative deviation were listed in Table II, and Figure 5 (Case 2) shows the adsorption isotherms of pure

TABLE III. Deviation of model prediction for mixed-gas adsorption.

\begin{tabular}{lccc} 
Substances & Case 1 ARD $(\%)$ & Case 2 ARD $(\%)$ & Using PSD ARD (\%) \\
\hline $\mathrm{CH}_{4}$ & 28.5 & 33.4 & 24.1 \\
$\mathrm{CO}_{2}$ & 13.5 & 20.5 & 12.7 \\
Total deviation & 21.0 & 27.0 & 18.4 \\
\hline
\end{tabular}



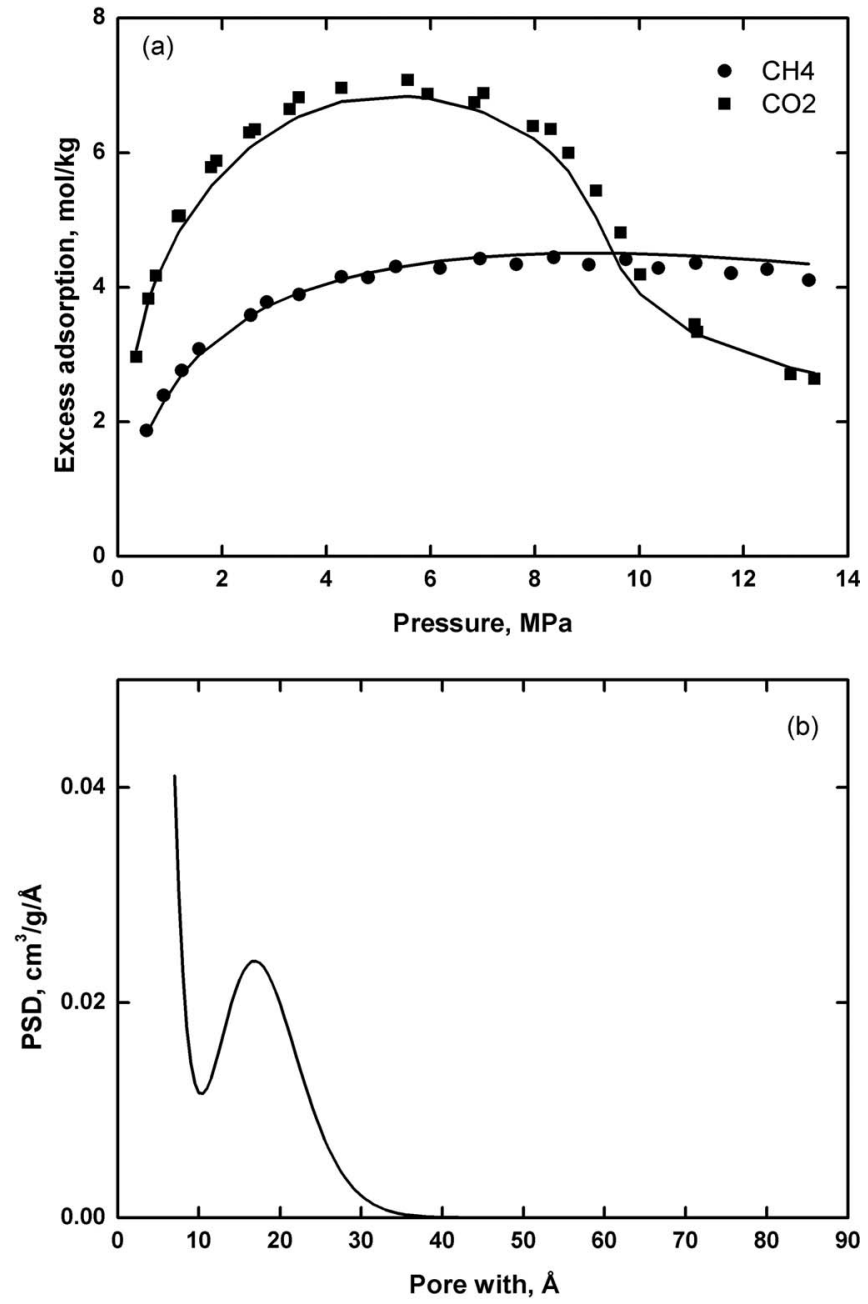

FIG. 7. (a) Methane and carbon dioxide adsorptions on Calgon F400 activated carbon with PSD. Symbols are experimental data $;{ }^{30}$ lines represent the results of the model. (b) PSD of Calgon F400 activated carbon.

gases. This model yields slightly better correlation results. Figure 6 (Case 2) shows the predicted adsorption isotherms of mixtures, which shows the similar prediction compared to those in Figure 6 (Case 1). The deviation of model prediction is listed in Table III. Considering the numerical simplicity, we prefer to calculate the weighted density by Eq. (16).

\section{Effect of pore size distribution on model performance}

As mentioned in the forgoing text that the pore size is a distribution instead of a single effective slit pore. To investigate the effect of pore size distribution (PSD) on model performance, the model was further extended to represent the gas adsorption with PSD.

With the consideration of PSD, the adsorption isotherm is expressed as ${ }^{31}$

$$
q(T, P)=\int_{H_{\min }}^{H_{\max }} q^{l o c a l}(T, P, H) f(H) d H,
$$

where $q(T, P)$ is the adsorption isotherm, $H_{\min }$ and $H_{\max }$ are the smallest and largest pores in PSD analysis, respectively, $q^{\text {local }}(T, P, H)$ is a local adsorption isotherm calculated by PC-SAFT-DFT in a pore width $H$ at fixed temperature and pressure, and $f(H)$ is the pore size distribution (PSD).

In this work, $f(H)$ was assumed as a bimodal gamma distribution due to its calculation simplicity, ${ }^{31}$ i.e.,

$$
f(H)=\sum_{i=1}^{2} \frac{\alpha_{i}\left(\gamma_{i} H\right)^{\beta_{i}}}{\Gamma\left(\beta_{i}\right) H} \exp \left(-\gamma_{i} H\right)
$$

where $\alpha_{i}, \beta_{i}$, and $\gamma_{i}$ are parameters.

For the studied activated carbon, PSD is not available. Therefore, in this work, the parameters of $\alpha_{i}, \beta_{i}$, and $\gamma_{i}$ in calculating $f(H)$ were determined from the fitting of experimental pure-gas adsorption data. In parameter fitting, the parameters of $\sigma_{s}$ and $\varepsilon_{s} / k$ were taken from literature ${ }^{32}\left(\sigma_{s}=3.4 \AA\right.$; $\left.\varepsilon_{s} / k=28\right)$, and the solid-fluid interaction was represented by Eq. (21) with $\rho_{\text {atom }}=0.382$ atoms $/ \AA^{2}$ and $\Delta=3.35 \AA$. The
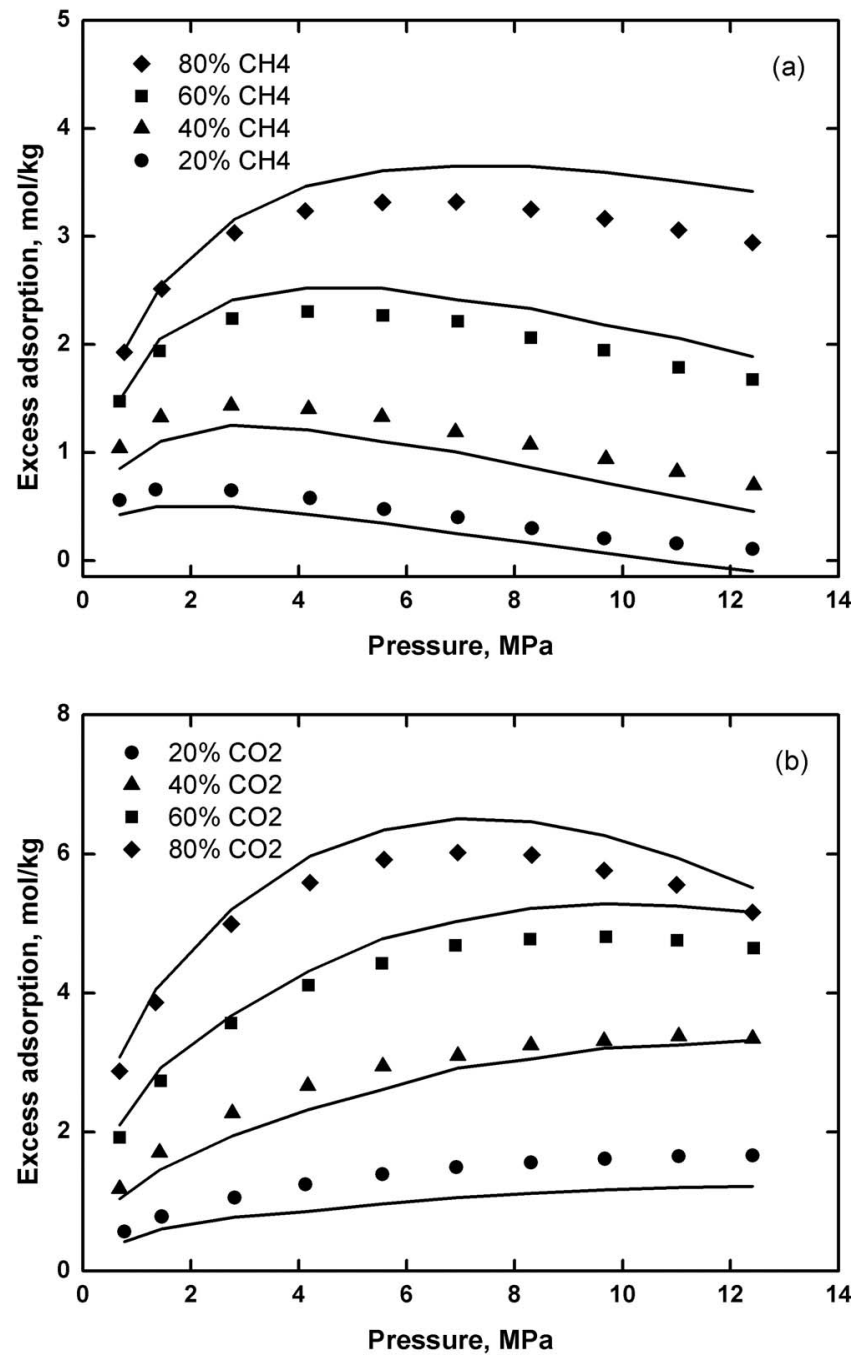

FIG. 8. Excess adsorption of $\mathrm{CH}_{4}$ (a) and $\mathrm{CO}_{2}$ (b) in $\mathrm{CH}_{4} / \mathrm{CO}_{2}$ mixtures on Calgon F400 activated carbon at $318.2 \mathrm{~K}$ with PSD. Symbols are experimental data $;{ }^{30}$ lines represent the results of the model. 
objective function $E$ was written as

$$
E=\frac{1}{N_{\text {exp }}} \sum_{i=1}^{N_{\text {exp }}}\left(\sum_{j=1}^{N_{H}} q^{\text {local }}\left(T, P_{i}, H_{j}\right) f\left(H_{j}\right) \Delta H-q^{\exp }\left(T, P_{i}\right)\right)^{2},
$$

where $N_{H}$ is the total number of pore widths.

The adsorption isotherms for both $\mathrm{CH}_{4}$ and $\mathrm{CO}_{2}$ were used to obtain the parameters of $\alpha_{i}, \beta_{i}$, and $\gamma_{i}$. The model performance and the corresponding PSD are illustrated in Figure 7. Compared to the results without the consideration of PSD shown in Figure 5, the model performance was improved, especially for $\mathrm{CO}_{2}$-adsorption isotherm.

The model with PSD was used to predict the mixed-gas adsorption. The model results are depicted in Figure 8 and the corresponding ARDs are listed in Table III. The model prediction is also improved compared to the case without PSD consideration. The deviation of the model prediction with PSD is down to $18.4 \%$, which is comparable to the results from other models. ${ }^{33}$

Therefore, with the consideration of PSD, the results of the model for both pure- and mixed-gas adsorption isotherms can be improved more or less. However, it will cost more computation time. Considering the results of Case 1 in III C are acceptable, we prefer to use the model without PSD.

\section{CONCLUSIONS}

In this work, we extended the developed PC-SAFT-DFT for pure fluid to mixtures. The model performance was verified by comparing the model prediction of the density profiles with molecular simulation results. The developed model was further used to represent the isotherm adsorption of methane and carbon dioxide on activated carbons, in which the pore of the activated carbon was modeled as a slit with a LennardJones potential interacting between slit surface and fluid and the parameters of methane and carbon dioxide were the same as those in the bulk PC-SAFT. Without any additional parameters the model can be used to predict the adsorption of methane/carbon dioxide mixtures, which illustrates the model's feasibility for representing the confined behaviors of real substances.

\section{ACKNOWLEDGMENTS}

X.J. and G.S. thank Swedish research council. G.S. and X.L. thank the Key Project of the National Natural Science
Foundation of China (Grant No. 21136004) and National Basic Research Program of China (Grant No. 2013CB733500). The computations were performed on resources provided by the Swedish National Infrastructure for Computing (SNIC) at High Performance Computing Center North (HPC2N).

${ }^{1}$ T. Koga, N. Jiang, P. Gin, M. K. Endoh, S. Narayanan, L. B. Lurio, and S. K. Sinha, Phys. Rev. Lett. 107, 225901 (2011).

${ }^{2}$ P. T. Cummings, H. Docherty, C. R. Iacovella, and J. K. Singh, AIChE J. 56, 842-848 (2010).

${ }^{3}$ J. T. Eijkel and A. Berg, Microfluid. Nanofluid. 1, 249-267 (2005).

${ }^{4}$ C. P. Emborsky, Z. Feng, K. R. Cox, and W. G. Chapman, Fluid Phase Equilib. 306, 15-30 (2011).

${ }^{5}$ E. Kierlik and M. L. Rosinberg, J. Chem. Phys. 100, 1716-1730 (1994).

${ }^{6}$ S. Tripathi and W. G. Chapman, Phys. Rev. Lett. 94, 087801 (2005).

${ }^{7}$ S. Tripathi and W. G. Chapman, J. Chem. Phys. 122, 094506 (2005).

${ }^{8}$ Y. X. Yu, G. H. Gao, and X. L. Wang, J. Phys. Chem. B 110, 14418-14425 (2006).

${ }^{9}$ M. Borowko, A. Patrykiejew, W. Rzysko, S. Sokolowski, and J. Ilnytskyi, J. Chem. Phys. 134, 044705 (2011).

${ }^{10}$ Y. X. Yu and J. Z. Wu, J. Chem. Phys. 117, 2368-2376 (2002).

${ }^{11}$ Y. X. Yu and J. Z. Wu, J. Chem. Phys. 116, 7094-7103 (2002).

${ }^{12}$ J. Gross, J. Chem. Phys. 131, 204705 (2009).

${ }^{13}$ A. L. Frischknecht, V. Padmanabhan, and M. E. Mackay, J. Chem. Phys. 136, 164904 (2012).

${ }^{14}$ X. F. Xu, D. E. Cristancho, S. Costeux, and Z. G. Wang, J. Chem. Phys. 137, 054902 (2012).

${ }^{15}$ J. Hu, J. Chen, and J. Mi, Ind. Eng. Chem. Res. 51, 1236-1243 (2012).

${ }^{16}$ F. Llovell, A. Galindo, F. J. Blas, and G. Jackson, J. Chem. Phys. 133, 024704 (2010).

${ }^{17}$ M. B. Oliveira, M. Domínguez-Pérez, M. G. Freire, F. Llovell, O. Cabeza, J. A. Lopes-da-Silva, L. F. Vega, and J. A. P. Coutinho, J. Phys. Chem. B 116, 12133-12141 (2012).

${ }^{18}$ G. L. Shen, X. Y. Ji, and X. H. Lu, J. Chem. Phys. 138, 224706 (2013).

${ }^{19}$ J. Gross and G. Sadowski, Fluid Phase Equilib. 168, 183-199 (2000).

${ }^{20}$ J. Gross and G. Sadowski, Ind. Eng. Chem. Res. 40, 1244-1260 (2001).

${ }^{21}$ Y. X. Yu and J. Z. Wu, J. Chem. Phys. 117, 10156-10164 (2002).

${ }^{22}$ Z. C. Ye, J. Cai, H. L. Liu, and Y. Hu, J. Chem. Phys. 123, 194902 (2005).

${ }^{23}$ B. Peng and Y. X. Yu, Langmuir 24, 12431-12439 (2008).

${ }^{24}$ B. Peng and Y.-X. Yu, J. Phys. Chem. B 112, 15407-15416 (2008).

${ }^{25}$ Y. X. Yu, J. Chem. Phys. 131, 024704 (2009).

${ }^{26}$ L. L. Lee, J. Chem. Phys. 135, 204706 (2011).

${ }^{27}$ A. Yethiraj and C. K. Hall, J. Chem. Phys. 91, 4827-4837 (1989).

${ }^{28}$ S. Zhang, J. Cai, H. Liu, and Y. Hu, Mol. Simul. 30, 143-147 (2004).

${ }^{29}$ S. A. Mohammad, A. Arumugam, R. L. Robinson, and K. A. M. Gasem, Energy Fuels 26, 536-548 (2012).

${ }^{30}$ M. Sudibandriyo, Z. Pan, J. E. Fitzgerald, R. L. Robinson, and K. A. M. Gasem, Langmuir 19, 5323-5331 (2003).

${ }^{31}$ C. Lastoskie, K. E. Gubbins, and N. Quirke, J. Phys. Chem. 97, 4786-4796 (1993).

${ }^{32}$ W. A. Steele, Surf. Sci. 36, 317-352 (1973).

${ }^{33}$ M. A. Monsalvo and A. A. Shapiro, Fluid Phase Equilib. 283, 56-64 (2009). 